\title{
Carbon X-ray absorption in the local ISM: Fingerprints in X-ray Novae spectra
}

\author{
Efraín Gatuzz, ${ }^{1,2 \star}$ J.-U. Ness, ${ }^{3 \star}$ T. W. Gorczyca, ${ }^{4}$ M. F. Hasoglu, ${ }^{5}$ \\ Timothy R. Kallman ${ }^{6}$ and Javier A. García ${ }^{7}$ \\ ${ }^{1}$ ESO, Karl-Schwarzschild-Strasse 2, D-85748 Garching bei München, Germany \\ ${ }^{2}$ Excellence Cluster Universe, Boltzmannstrasse 2, D-85748, Garching, Germany \\ ${ }^{3}$ XMM-Newton Observatory SOC, European Space Astronomy Centre, Camino Bajo del Castillo s/n, Urb. Villafranca del Castillo, E-28692 Villanueva de la \\ Cañada, Madrid, Spain \\ ${ }^{4}$ Western Michigan University, Kalamazoo, MI 49008, USA \\ ${ }^{5}$ Department of Computer Engineering, Hasan Kalyoncu University, 27100 Sahinbey, Gaziantep, Turkey \\ ${ }^{6}$ NASA Goddard Space Flight Center, Greenbelt, MD 20771, USA \\ ${ }^{7}$ Cahill Center for Astronomy and Astrophysics, California Institute of Technology, Pasadena, CA 91125, USA
}

Accepted 2018 June 4. Received 2018 June 4; in original form 2018 February 1

\begin{abstract}
We present a study of the C K-edge using high-resolution LETGS Chandra spectra of four novae during their super-soft-source (SSS) phase. We identified absorption lines due to C II $\mathrm{K} \alpha, \mathrm{C}_{\mathrm{III}} \mathrm{K} \alpha$, and $\mathrm{C}$ III $\mathrm{K} \beta$ resonances. We used these astronomical observations to perform a benchmarking of the atomic data, which involves wavelength shifts of the resonances and photoionization cross-sections. We used improved atomic data to estimate the $\mathrm{C}_{\text {II }}$ and $\mathrm{C}$ III column densities. The absence of physical shifts for the absorption lines, the consistence of the column densities between multiple observations, and the high temperature required for the SSS nova atmosphere modelling support our conclusion about an interstellar medium origin of the respective absorption lines. Assuming a collisional ionization equilibrium plasma the maximum temperature derived from the ratio of $\mathrm{C}$ II/ $\mathrm{C}$ III column densities of the absorbers correspond to $T_{\max }<3.05 \times 10^{4} \mathrm{~K}$.
\end{abstract}

Key words: ISM: atoms-ISM: structure-X-rays: ISM.

\section{INTRODUCTION}

High-resolution X-ray spectroscopy constitutes a powerful technique to study the elements associated with the local interstellar medium (ISM), defined as gas and dust between the stars. Using an $\mathrm{X}$-ray bright source, acting as a lamp, the absorption features identified in the X-ray spectra provide information about the physical properties of the gas between the source and the observer. Using $\mathrm{X}$-ray spectra of low-mass $\mathrm{X}$-ray binaries, the $\mathrm{O}, \mathrm{Fe}, \mathrm{Ne}, \mathrm{Mg}$, and $\mathrm{Si} \mathrm{K}$ absorption edges associated with the ISM have been analysed in previous works (Juett, Schulz \& Chakrabarty 2004; Ueda et al. 2005; Juett et al. 2006; Yao et al. 2009; Pinto et al. 2010; Costantini et al. 2012; Pinto et al. 2013; Gatuzz et al. 2013a,b; Liao, Zhang \& Yao 2013; Luo \& Fang 2014; Gatuzz et al. 2014, 2015; Gatuzz et al. 2016; Joachimi et al. 2016; Schulz, Corrales \& Canizares 2016; Nicastro et al. 2016a,b; Gatuzz \& Churazov 2018).

The ISM is composed of multiple phases, which depend on their characteristic temperatures and densities. Carbon, which consti-

\footnotetext{
^E-mail: efraingatuzz@gmail.com, egatuzzs@eso.org (EG);
} juness@sciops.esa.int (J-UN) tutes the fourth most abundant element in the Galaxy, can be used to probe the link between the different phases. $\mathrm{C}_{\mathrm{I}}$, for example, has been used to analyse the cold Galactic gas that is characterized by a relatively low thermal pressure using the Space Telescope Imaging Spectrograph on board the Hubble Space Telescope (Jenkins \& Tripp 2001; Burgh, France \& Jenkins 2010; Jenkins \& Tripp 2011), while the C II $158 \mu \mathrm{m}$ line allows the characterization of the cold atomic clouds in transition from an atomic to molecular form (Pineda et al. 2013, 2017; Langer et al. 2014; Pineda, Langer \& Goldsmith 2014; Richter et al. 2017; Savage et al. 2017). Also, it has been shown that solids that contain carbon atoms, such as graphite and polycyclic aromatic hydrocarbons, may constitute the main heat source for the ISM (Draine \& Li 2001; Helou et al. 2001; Okada et al. 2013; Chen, Li \& Zhang 2017; Shannon et al. 2018). In this sense, it is essential to estimate the amount of $\mathrm{C}$ depleted in the dust phase in order to fully understand the heating-cooling ISM processes.

One of the advantages of the high-resolution X-ray spectroscopy is that it provides access to both gas and solid components of the ISM. The C K-edge, located at 38-44 ̊ wavelength, can be accessed only through the low-energy transmission grating (LETG) on board 
Table 1. List of Chandra LETGS-HRC observations.

\begin{tabular}{|c|c|c|c|c|c|c|}
\hline Source & ObsID. & Obs. date & $\begin{array}{l}\text { Exp. time } \\
\text { (ks) }\end{array}$ & $\begin{array}{c}N(\mathrm{HI}) \\
\left(10^{21} \mathrm{~cm}^{-2}\right)\end{array}$ & $\begin{array}{l}\text { count-rate } \\
\text { (counts/s) }\end{array}$ & $\begin{array}{c}\text { Counts } \\
(38-44 \AA)\end{array}$ \\
\hline \multirow[t]{3}{*}{ KTEri } & 12097 & $23-01-2010$ & 14.9 & \multirow[t]{3}{*}{0.52} & 11.52 & 25028 \\
\hline & 12101 & 06-02-2010 & 47.8 & & 37.48 & 16971 \\
\hline & 12203 & 21-04-2010 & 32.4 & & 106.5 & 26717 \\
\hline Sgr2015b & 16690 & $16-10-2015$ & 48 & 1.11 & 12.07 & 106572 \\
\hline & 15743 & $06-12-2013$ & 49 & 1.23 & 48.34 & 293788 \\
\hline \multirow[t]{3}{*}{ V4743Sgr } & 3775 & $19-03-2003$ & 20.3 & \multirow[t]{3}{*}{1.05} & 38.91 & 45173 \\
\hline & 3776 & $18-07-2003$ & 11.7 & & 37.16 & 26349 \\
\hline & 4435 & $25-09-2003$ & 12.0 & & 19.78 & 16209 \\
\hline
\end{tabular}

Note: $N(\mathrm{HI})$ column densities obtained from Kalberla et al. (2005).

Table 2. Absorption line assignments with observed wavelength.

\begin{tabular}{|c|c|c|c|c|c|c|}
\hline \multirow[t]{2}{*}{ Source } & \multirow[t]{2}{*}{ ObsID. } & \multicolumn{3}{|c|}{ CII } & \multicolumn{2}{|c|}{ CIII } \\
\hline & & $K \alpha_{1}(\AA)$ & $K \alpha_{2}(\AA)$ & $K \alpha_{3}(\AA)$ & $K \alpha(\AA)$ & $K \beta(\AA)$ \\
\hline Theoretical & & 43.0592 & 42.9867 & 42.6818 & 42.2360 & 38.4459 \\
\hline & 12100 & $43.0736 \pm 0.0039$ & $43.0005 \pm 0.0039$ & $42.7863 \pm 0.0039$ & - & - \\
\hline & 12101 & $43.0623 \pm 0.0047$ & $42.9875 \pm 0.0047$ & $42.7626 \pm 0.0047$ & $42.2042 \pm 0.0046$ & - \\
\hline & 12203 & $43.0725 \pm 0.0047$ & $43.0002 \pm 0.0047$ & $42.7748 \pm 0.0047$ & - & - \\
\hline \multirow[t]{2}{*}{ V339Del } & 15742 & $43.0703 \pm 0.0043$ & $42.9875 \pm 0.0043$ & $42.7707 \pm 0.0043$ & $42.1873 \pm 0.0042$ & $38.4093 \pm 0.0038$ \\
\hline & 15743 & $43.0642 \pm 0.0047$ & $42.9906 \pm 0.0047$ & $42.7800 \pm 0.0047$ & $42.1752 \pm 0.0046$ & $38.4082 \pm 0.0042$ \\
\hline \multirow[t]{3}{*}{ V4743Sgr } & 3775 & $43.0725 \pm 0.0039$ & $42.9847 \pm 0.0039$ & $42.7666 \pm 0.0038$ & $42.1878 \pm 0.0038$ & $38.4039 \pm 0.0035$ \\
\hline & 3776 & $43.0684 \pm 0.0043$ & $42.9869 \pm 0.0043$ & $42.7674 \pm 0.0043$ & $42.1632 \pm 0.0042$ & - \\
\hline & 4435 & $43.0684 \pm 0.0043$ & $42.9795 \pm 0.0043$ & $42.7748 \pm 0.0043$ & $42.1615 \pm 0.0042$ & $38.4133 \pm 0.0038$ \\
\hline Average & & $43.0694 \pm 0.0074$ & $42.9851 \pm 0.0074$ & $42.7751 \pm 0.0074$ & $42.1840 \pm 0.0043$ & $38.4089 \pm 0.0038$ \\
\hline
\end{tabular}

of the Chandra observatory. Super-soft-sources (SSS) provide highcount continuum spectra that can be used to perform such analysis. Although their X-ray spectra can be complex, showing spectral features due to multiple temperature components, carbon absorption features have been identified (Ness et al. 2009; Rauch et al. 2010; van Rossum 2012; Rauch 2016).

In this paper, we present an analysis of the $\mathrm{C}$ absorption features in the ISM using Chandra high-resolution spectra of four sources. The outline of this paper is as follows: In Section 2 we describe the observations and data reduction. In Section 3 we describe the $\mathrm{C}$ K-edge modelling and the atomic data involved. In Section 4 we discuss the main results. Finally, in Section 5 we summarize our main conclusions.

\section{OBSERVATIONS AND DATA REDUCTION}

We analyse Chandra spectra of four SSS in order to study the ISM carbon K-edge along different lines of sight. Because of their brightness and proximity, SSS high-resolution spectra constitute a useful way to analyse not only the binary system involved but also the ISM fingerprints identified as absorption features. Table 1 lists the specifications for each LETGS-HRC observation, the observation date, the exposure time, the hydrogen column density $21 \mathrm{~cm}$ measurement from the Kalberla et al. (2005) survey, the count rate, and the number of counts in the C K- $\alpha$ wavelength region (38-44 $\AA$ ). The data were reduced using the Chandra Interactive Analysis of Observations software (CIAO,version 4.9) and following the standard procedure to obtain the LETG spectra. ${ }^{1}$ For each observation we combine the $+1 /-1$ orders using the combine_grating_spectra script. We use the XSPEC analysis data package (version 12.9.12, Arnaud 1996) to perform the spectral fitting. Finally, we use $\chi^{2}$ statistic with the weighting method for low counts regime defined by Churazov et al. (1996)

\section{C K-EDGE MODELLING}

In order to analyse the $\mathrm{C}$ K-edge absorption region (38-44 $\AA$ ), we first used a functional model consisting of a power-law continuum with absorption lines described by Gaussian profiles. Table 2 shows the wavelength position for all absorption lines identified in the spectra as well as their average values. Theoretical values obtained

\footnotetext{
${ }^{1}$ http://cxc.harvard.edu/ciao/threads/gspec.html

${ }^{2}$ https://heasarc.gsfc.nasa.gov/xanadu/xspec/
} 

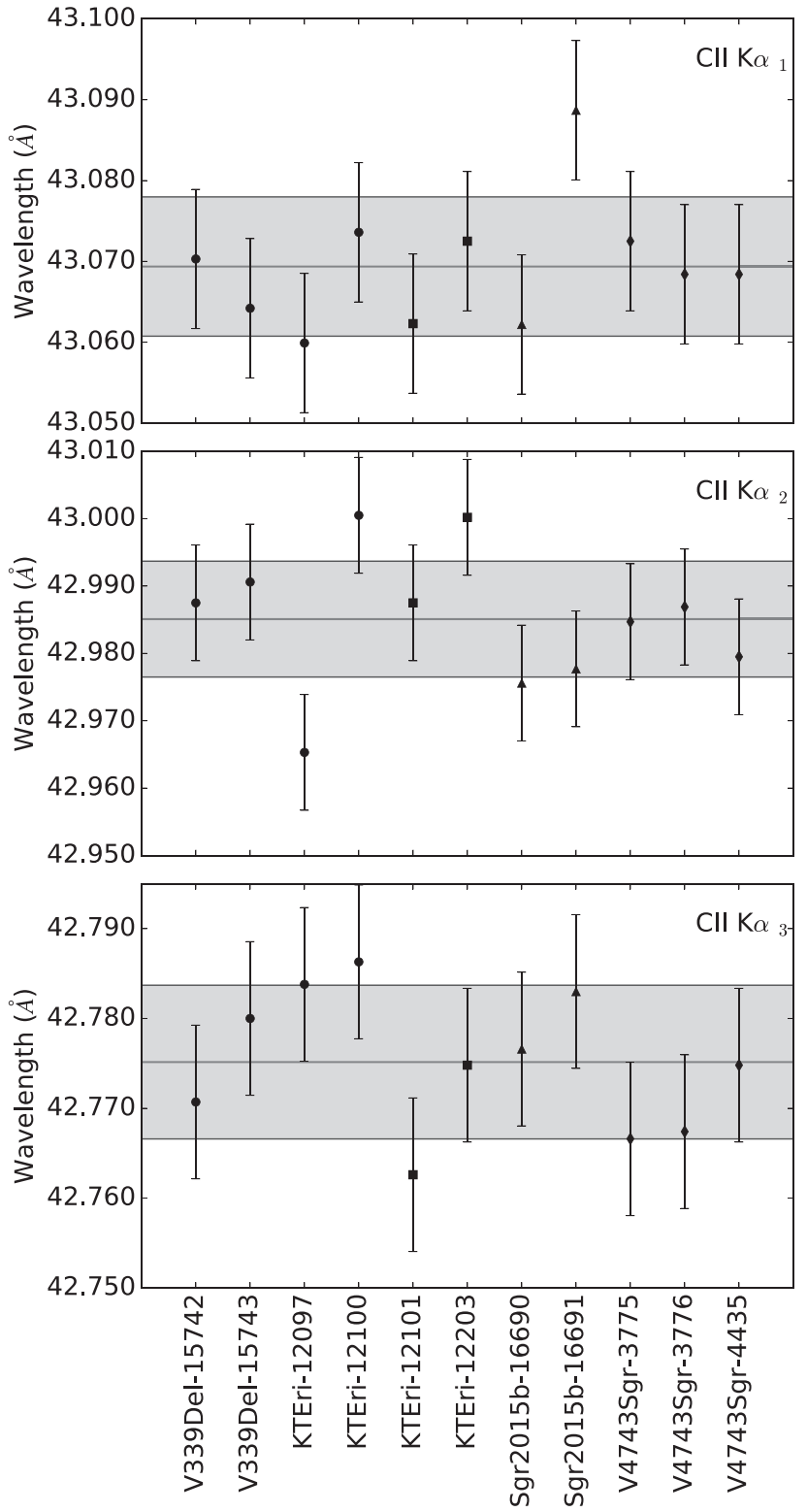

Figure 1. $\mathrm{C}_{\text {II }} \mathrm{K} \alpha$ resonances observational wavelengths determined from Gaussian fits. The horizontal grey region correspond to the average value and its 90 per cent uncertainty.

from Hasoglu et al. (2010) calculations are also listed. Both cameras, the High Resolution Camera (HRC) and the Advanced CCD Imaging Spectrometer (ACIS), have $\mathrm{C}_{\mathrm{I}}$ instrumental absorption at $\sim 43.6 \AA$ due to absorption edges in the materials comprising the instruments, as indicated by the Chandra Proposers' Observatory Guide. ${ }^{3}$ We have not identified $\mathrm{C}_{\mathrm{I}}$ absorption lines in excess of instrumental features that could be associated with the ISM in the $\mathrm{X}$-ray spectra sample. In this sense, it has been shown that the $\mathrm{C}_{\mathrm{I}}$ column density associated with the ISM tends to be lower than $10^{14}$ $\mathrm{cm}^{-2}$ along multiple lines of sight, including regions with large $\mathrm{H} \mathrm{I}$

\footnotetext{
${ }^{3}$ http://cxc.harvard.edu/proposer/POG/html/index.html
}

column densities (Jenkins \& Tripp 2011; Gerin et al. 2015; Welty et al. 2016; Pineda et al. 2017).

Fig. 1 shows the wavelength positions for each resonance in the $\mathrm{C}$ II $\mathrm{K} \alpha$ triplet, which have been measured in all observations. It is important to note that, considering the uncertainties, the wavelength positions tend to agree not only between different observations of the same source but also for different sources. $\mathrm{C}$ III $\mathrm{K} \alpha$ and $\mathrm{C}$ III $\mathrm{K} \beta$ absorption lines were identified in seven and five observations, respectively. Fig. 2 shows the best-fitting wavelength positions measured for both resonances. In both figures the horizontal grey regions indicate the average values and their uncertainties. It is clear from the plots that, considering the uncertainties, the wavelength positions do not show significant shift between observations.

We performed a benchmarking of the atomic data by comparing the observed and theoretical absorption lines in the $\mathrm{C}$ K-edge region. Left-hand panel in Fig. 3 shows the $\mathrm{C}_{\mathrm{I}}$ (black line), $\mathrm{C}_{\text {II }}$ (red line), and $\mathrm{C}$ III (blue line) photoabsorption cross-sections computed by Hasoglu et al. (2010). Vertical lines correspond to the average measurements listed in Table 1. It is clear that the resonance positions for the $\mathrm{K} \alpha$ transitions differ between the theoretical predictions and the observational measurements. In this sense we have adjusted cross-sections in order to obtain the best possible agreement with the observed lines. The shifts are $+10.2 \mathrm{~m} \AA,-1.59 \mathrm{~m} \AA$, and $+93.3 \mathrm{~m} \AA$ for the $\mathrm{C}$ II $\mathrm{K} \alpha_{1}, \mathrm{~K} \alpha_{2}$, and $\mathrm{K} \alpha_{3}$ resonances, respectively. The larger shift for the $\mathrm{K} \alpha_{3}$ is expected because the $n=3$ resonances carry the greatest energy uncertainty along a Rydberg series (Hasoglu et al. 2010). For C III we move the $\mathrm{K} \alpha$ and the whole cross-section by $-52 \mathrm{~m} \AA$ and $-37 \mathrm{~m} \AA$, respectively. From the theoretical point of view, a $\sim 59 \mathrm{~m} \AA$ underprediction in wavelength is expected (Hasoglu et al. 2010). Right-hand panel in Fig. 3 shows the cross-sections after the wavelength corrections. It is important to mention that such benchmarking has been performed previously for the oxygen and neon photoabsorption cross-sections by Gatuzz et al. (2013a,b, 2015) concluding that the Chandra wavelength calibration can be safely used to correct the theoretical wavelength resonance positions.

\section{RESULTS AND DISCUSSIONS}

We included the corrected cross-sections from Hasoglu et al. (2010) in the ISMabs ${ }^{4}$ model in order to estimate $\mathrm{C}$ II and $\mathrm{C}$ III column densities in the SSS spectra listed in Table 1. Fig. 4 shows the best-fitting results using the ISMabs model for the $\mathrm{C}_{\text {II }} \mathrm{K}-\alpha$ wavelength region (42.5-43.3 $\AA$ ). In each panel, the black data points correspond to the observation. The model before the atomic data corrections and after the correction is indicated (red dashed and blue solid lines, respectively). In all cases we obtained a data-fitting improvement of $\Delta \chi^{2}>20$. Table 3 lists the column densities obtained for each observation. Due to the elemental abundance enrichments in the ejected material from the respective novae explosion through the mixing process (Kelly et al. 2013), differences between column densities for observations performed at different epochs are expected if the absorbing material is intrinsic to the source. Fig. 5 shows a comparison between the column densities obtained from the ISMabs model. It is clear from the plot that the column densities tend to agree for different observations of the same source. Differences between sources, on the other hand, are expected due

\footnotetext{
${ }^{4}$ https://heasarc.gsfc.nasa.gov/xanadu/xspec/models/ismabs.html
} 

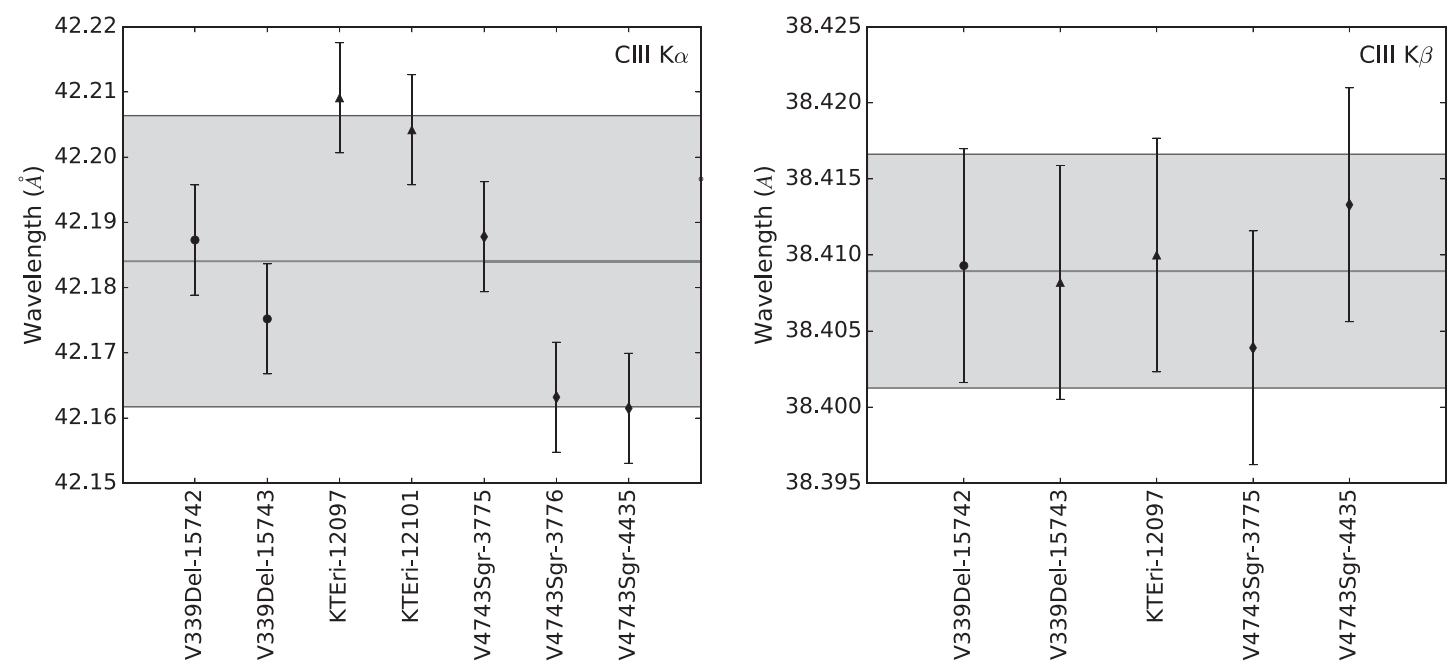

Figure 2. Left-hand panel: $\mathrm{C}$ III $\mathrm{K} \alpha$ resonance observational wavelengths determined from Gaussian fits. Right-hand panel: $\mathrm{C}$ III $\mathrm{K} \beta$ resonance observational wavelengths determined from Gaussian fits. The horizontal grey region corresponds to the average value and its 90 per cent uncertainty.
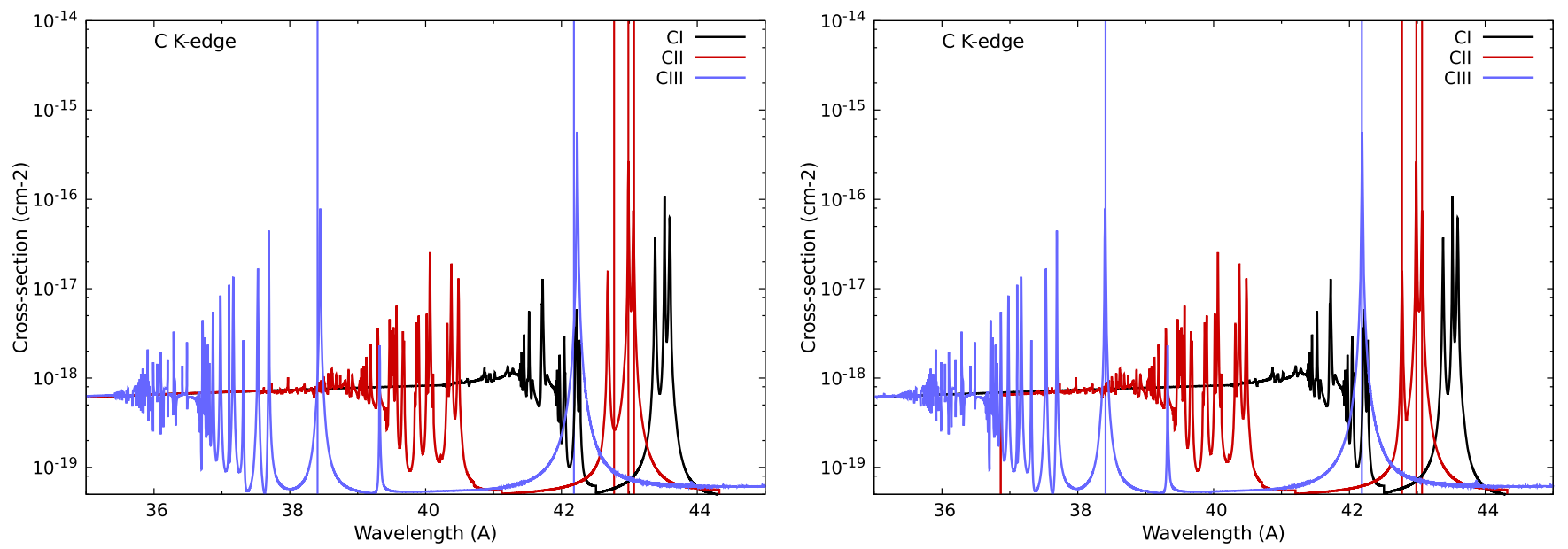

Figure 3. C I, C II, and C III photoabsorption cross-sections computed by Hasoglu et al. (2010) that are implemented in the ISMabs model. Vertical dashed lines correspond to the average measurements listed in Table 1. Left-hand panel displays the original cross-sections while right-hand panel shows the same curves after the benchmarking.

to the density distribution of the ISM gas along the Galaxy (Robin et al. 2003; Kallman et al. 2009; Nicastro et al. 2016a,b; Gatuzz \& Churazov 2018).

Another possibility is an origin in the SSS atmosphere that usually shows multiple absorption features (Ness 2012; Orio 2012; Ness et al. 2013). However, such atmospheres require high temperatures ( $>0.6 \mathrm{MK}$ ), for which we will not find $\mathrm{C}_{\mathrm{I}}, \mathrm{C}$ II, and $\mathrm{C}$ III ions (Ness et al. 2009; Rauch et al. 2010; van Rossum 2012; Rauch 2016). It is important to compare the one to two months observation separation time in our sample with the novae evolution time-scale, which can vary from months to years (Schwarz et al. 2011). For example, Ness et al. (2007) modelled the X-ray high-resolution spectra of the RS Ophiuchi novae, a source that shows a notable evolution in both, the continuum and the emission/absorption features, within months (see fig. 1 in Ness et al. 2009). The model used by Ness et al. (2007) included a component for the ISM local absorption and a second component to model the circumstellar material intrinsic to the source. The best-fitting requires that the oxygen contribution from the circumstellar material disappears around day 54 after the outburst, probably due to photoionization of the local gas by the radiation field.

Fig. 6 shows a comparison between $\mathrm{C}$ II $\mathrm{K} \alpha_{1}$ (top pannels) and $\mathrm{C}$ vi $\mathrm{K} \alpha$ (bottom pannels) absorption lines parametrized in velocity space for each source analysed in this work. The $\mathrm{C}$ VI $\mathrm{K} \alpha$ absorption line has been identified as intrinsic to the source previously (Ness et al. 2003; Petz et al. 2005; Ness et al. 2007; Ness 2012; van Rossum 2012). It is clear from the plot that the $\mathrm{C}_{\text {II }} \mathrm{K} \alpha_{1}$ remains at the same wavelength while there are no $\mathrm{C}$ VI $\mathrm{K} \alpha$ absorption features at rest wavelength. Also, some high-resolution X-ray novae spectra have shown P Cygni profiles (Ness et al. 2007; Orio et al. 2013) that we have not identified in the analysed spectra. 

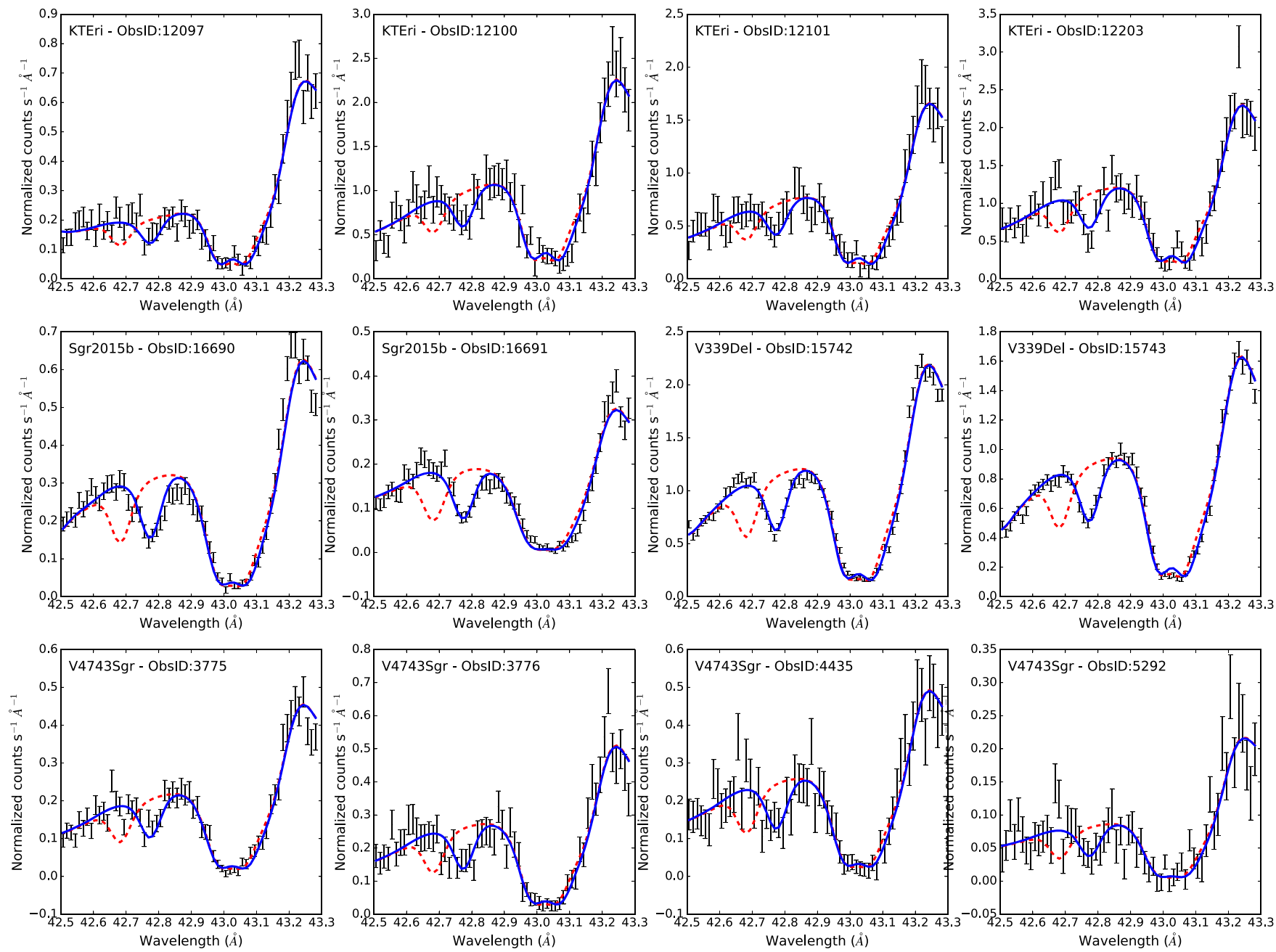

Figure 4. Best-fitting results using Chandra LETG data for the $\mathrm{C}$ II $\mathrm{K}$-edge wavelength region. The lines correspond to the model before and after the atomic data benchmarking (red dashed and blue solid lines, respectively).

It is important to note that there is no reliable method to determine the total amount of carbon emitted in X-ray ejecta, in order to compare with the ISM abundance. Theoretical estimation, such as Rauch et al. (2010), depends on multiple factors including the composition of the accreted material, nuclear burning products, composition of the white dwarf, and the amount of mixing of white dwarf material into the ejecta.

Table 3 also lists the $\mathrm{C}$ II/C III ratios for those sources for which both column densities can be estimated. It is clear that $\mathrm{C}$ II dominates in all cases. In this sense, previous analysis using Herschel Galactic observations show that $\mathrm{C}$ II constitutes the main carbon reservoir along the lines of sight in most cases (Pineda et al. 2014; Gerin et al. 2015). The ion fractions depend on the physical state of the plasma. We used the $\operatorname{XSTAR}^{5}$ code to estimate the maximum temperature of the gas assuming collisional ionization equilibrium (see Gatuzz \& Churazov 2018). Fig. 7 shows the $\mathrm{C}$ II/C III ratio obtained from the XSTAR calculations. Horizontal dashed lines correspond to the ratios listed in Table 3 while the vertical arrow indicates the maximum temperature derived. We found $T_{\max }<3.05 \times 10^{4} \mathrm{~K}$.
Table 3. Column density best-fitting results.

\begin{tabular}{lcccc}
\hline Source & ObsID. & $N(\mathrm{CII})$ & $N(\mathrm{CIII})$ & $\mathrm{CII} / \mathrm{CIII}$ \\
\hline KTEri & 12097 & $18.63_{-2.55}^{+2.99}$ & $0.58_{-0.38}^{+0.58}$ & $32.12 \pm 27.00$ \\
& 12100 & $19.51_{-2.81}^{+3.34}$ & & \\
& 12101 & $20.81_{-3.10}^{+3.73}$ & & \\
Sgr2015b & 12203 & $20.36_{-2.65}^{+3.11}$ & & \\
& 16690 & $33.50_{-2.19}^{+2.43}$ & & \\
V339Del & 16691 & $35.06_{-3.66}^{+4.27}$ & & \\
& 15742 & $24.98_{-0.83}^{+0.87}$ & $0.53_{-0.13}^{+0.14}$ & $47.13 \pm 12.11$ \\
V4743Sgr & 15743 & $22.91_{-0.88}^{+0.92}$ & $0.65_{-0.15}^{+0.16}$ & $35.24 \pm 8.51$ \\
& 3775 & $32.28_{-2.36}^{+3.36}$ & & \\
& 3776 & $29.50_{-3.81}^{+4.64}$ & $1.26_{-0.46}^{+0.58}$ & $23.41 \pm 10.24$ \\
& 4435 & $31.19_{-4.98}^{+6.40}$ & $0.89_{-0.60}^{+0.95}$ & $35.04 \pm 31.37$ \\
\hline
\end{tabular}

Note: Column densities in units of $10^{16} \mathrm{~cm}^{-2}$. 

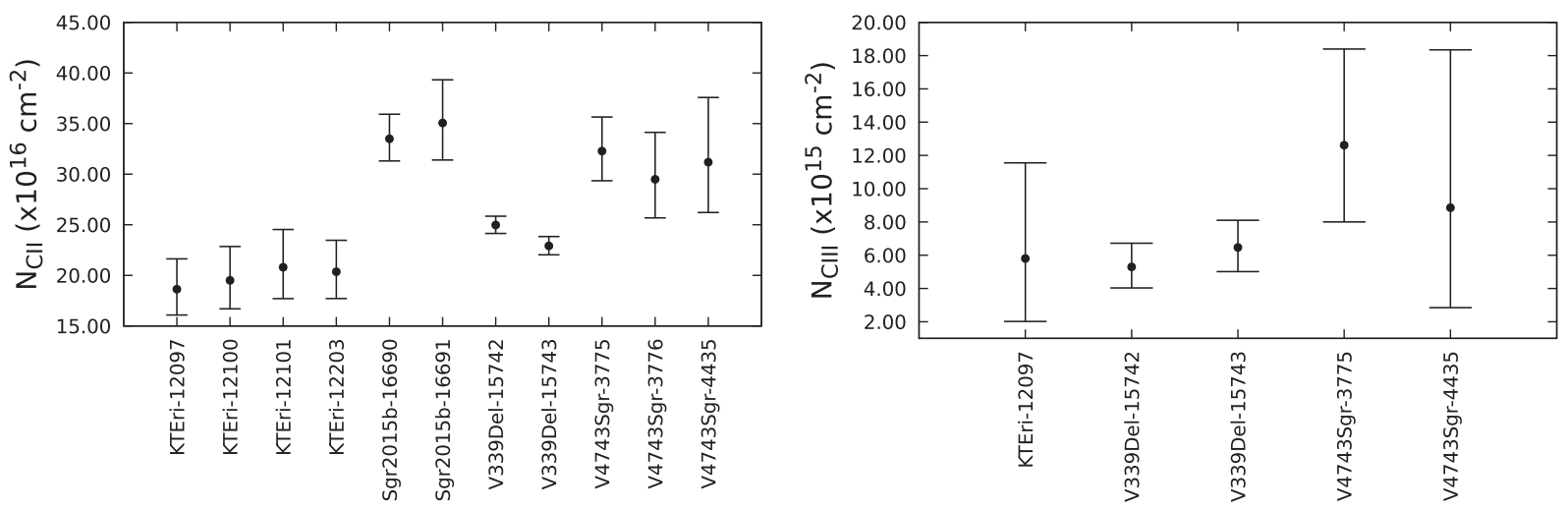

Figure 5. Left-hand pannel: $\mathrm{C}_{\text {II }}$ column densities obtained from the best ISMabs fit. Right-hand pannel: C III column densities obtained from the best ISMabs fit.
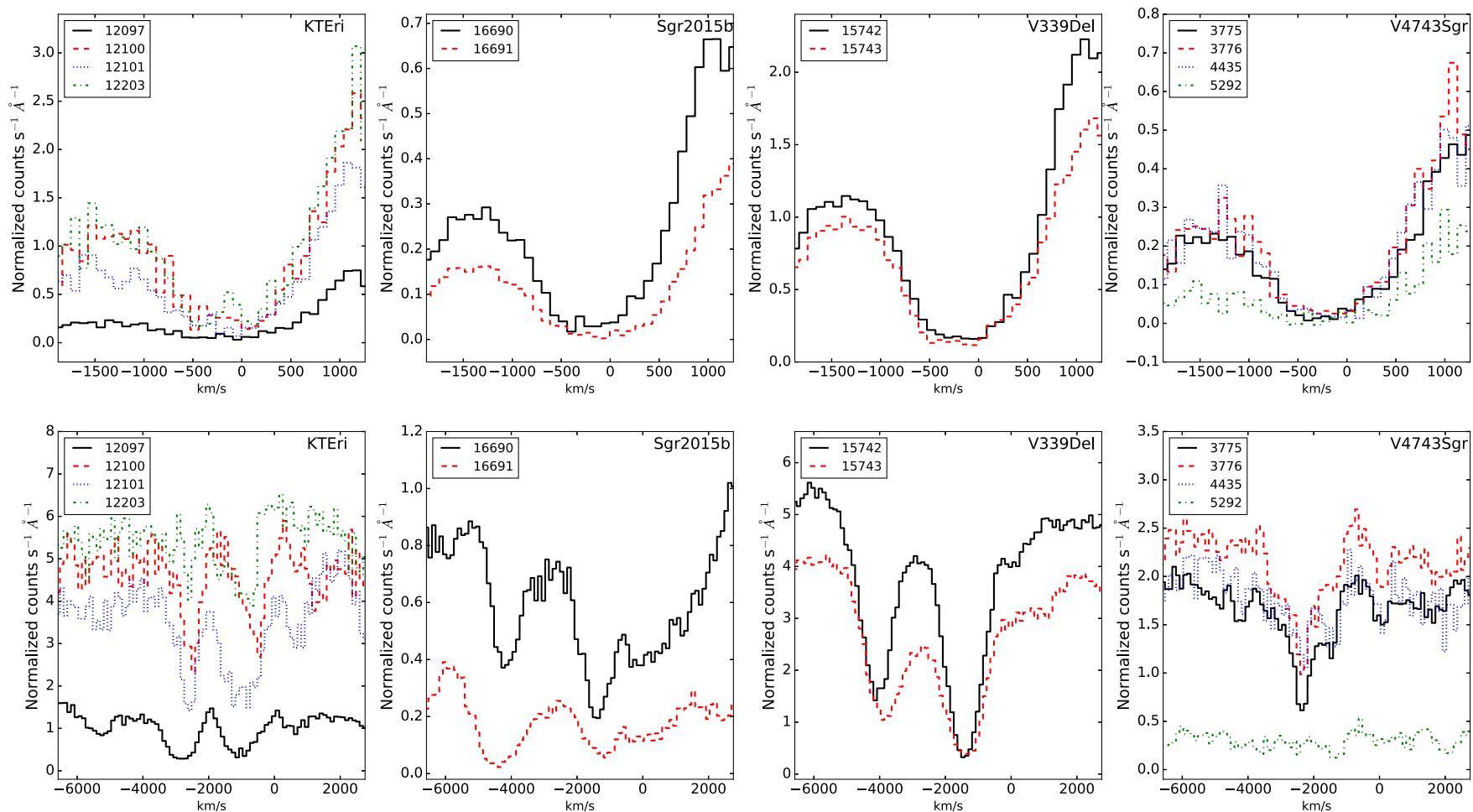

Figure 6. $\mathrm{C}$ II $\mathrm{K} \alpha_{1}$ (top pannels) and $\mathrm{C}$ vi $\mathrm{K} \alpha$ (bottom pannels) absorption lines parametrized in velocity space for each source analysed.

\section{CONCLUSIONS AND SUMMARY}

We have performed an analysis of the C K-edge using highresolution Chandra spectra of four SSS. The instrumental features due to a $\mathrm{C}$ layer in the camera prevent the analysis of $\mathrm{C}_{\mathrm{I}}$ absorption. We have detected all three resonances of the $\mathrm{C}$ II $\mathrm{K} \alpha$ in 11 observations as well as the $\mathrm{C}$ III $\mathrm{K} \alpha$ and $\mathrm{K} \beta$ in seven and five observations, respectively. We used the astronomical observations in order to perform a benchmarking of the atomic data computed by Hasoglu et al. (2010). We have included these corrected crosssections in the ISMabs X-ray absorption model. Using the improved atomic data we estimated the $\mathrm{C}_{\mathrm{II}}$ and $\mathrm{C}$ III column densities for each observation. While high-ionized lines such as $\mathrm{C}$ VI $\mathrm{K} \alpha$ show significant shifts between different observations and different sources, the $\mathrm{C}$ II and $\mathrm{C}$ III wavelength positions are consistent.
The absence of physical shifts for the absorption lines, the lack of variability for the column densities among different observations, and the low temperatures associated with these ions compared to Xray novae typical atmosphere temperature support our conclusion about an ISM origin of the absorption lines identified in the spectra. From the ratios of $\mathrm{C}$ II/C III column densities, we found $T_{\max }<$ $3.05 \times 10^{4} \mathrm{~K}$ that corresponds to the so-called warm component of the ISM.

\section{ACKNOWLEDGEMENTS}

We are grateful to the referee, Dr. Lia Corrales, for the careful reading of our manuscript and the valuable comments that led to improvements of its scientific content. 


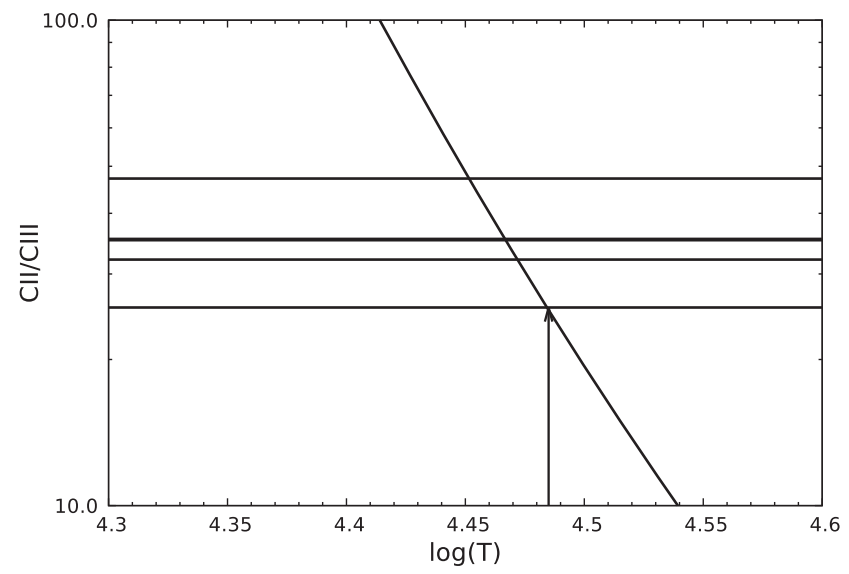

Figure 7. $\mathrm{C}$ II/ $\mathrm{C}$ III ratio obtained from XSTAR calculations assuming collisional ionization equilibrium plasma. Horizontal dashed lines correspond to the ratios listed in Table 3. The arrow indicates the maximum temperature obtained.

\section{REFERENCES}

Arnaud K. A., 1996, in Jacoby G. H., Barnes J., eds, ASP Conf. Ser. 101, San Francisco, p.17

Burgh E. B., France K., Jenkins E. B., 2010, ApJ, 708, 334

Chen X. H., Li A., Zhang K., 2017, ApJ, 850, 104

Churazov E., Gilfanov M., Forman W., Jones C., 1996, ApJ, 471, 673

Costantini E. et al., 2012, A\&A, 539, A32

Draine B. T., Li A., 2001, ApJ, 551, 807

Gatuzz E., Churazov E., 2018, MNRAS, 474, 696

Gatuzz E. et al., 2013a, ApJ, 768, 60

Gatuzz E. et al., 2013b, ApJ, 778, 83

Gatuzz E., García J., Mendoza C., Kallman T. R., Bautista M. A., Gorczyca T. W., 2014, ApJ, 790, 131

Gatuzz E., García J., Kallman T. R., Mendoza C., Gorczyca T. W., 2015, ApJ, 800, 29

Gatuzz E., García J. A., Kallman T. R., Mendoza C., 2016, A\&A, 588, A111

Gerin M. et al., 2015, A\&A, 573, A30

Hasoglu M. F., Abdel-Naby S. A., Gorczyca T. W., Drake J. J., McLaughlin B. M., 2010, ApJ, 724, 1296

Helou G., Malhotra S., Hollenbach D. J., Dale D. A., Contursi A., 2001, ApJ, 548, L73

Jenkins E. B., Tripp T. M., 2001, ApJS, 137, 297

Jenkins E. B., Tripp T. M., 2011, ApJ, 734, 65

Joachimi K., Gatuzz E., García J. A., Kallman T. R., 2016, MNRAS, 461, 352

Juett A. M., Schulz N. S., Chakrabarty D., 2004, ApJ, 612, 308
Juett A. M., Schulz N. S., Chakrabarty D., Gorczyca T. W., 2006, ApJ, 648, 1066

Kalberla P. M. W., Burton W. B., Hartmann D., Arnal E. M., Bajaja E., Morras R., Pöppel W. G. L., 2005, A\&A, 440, 775

Kallman T. R., Bautista M. A., Goriely S., Mendoza C., Miller J. M., Palmeri P., Quinet P., Raymond J., 2009, ApJ, 701, 865

Kelly K. J., Iliadis C., Downen L., José J., Champagne A., 2013, ApJ, 777, 130

Langer W. D., Velusamy T., Pineda J. L., Willacy K., Goldsmith P. F., 2014, A\&A, 561, A122

Liao J.-Y., Zhang S.-N., Yao Y., 2013, ApJ, 774, 116

Luo Y., Fang T., 2014, ApJ, 780, 170

Ness J. U., 2012, Bull. Astron. Soc. India, 40, 353

Ness J.-U. et al., 2003, ApJ, 594, L127

Ness J.-U. et al., 2007, ApJ, 665, 1334

Ness J.-U. et al., 2009, AJ, 137, 3414

Ness J.-U. et al., 2013, A\&A, 559, A50

Nicastro F., Senatore F., Gupta A., Guainazzi M., Mathur S., Krongold Y., Elvis M., Piro L., 2016a, MNRAS, 457, 676

Nicastro F., Senatore F., Gupta A., Mathur S., Krongold Y., Elvis M., Piro L., 2016b, MNRAS, 458, L123

Okada Y. et al., 2013, A\&A, 553, A2

Orio M., 2012, Bull. Astron. Soc. India, 40, 333

Orio M. et al., 2013, MNRAS, 429, 1342

Petz A., Hauschildt P. H., Ness J.-U., Starrfield S., 2005, A\&A, 431, 321

Pineda J. L., Langer W. D., Velusamy T., Goldsmith P. F., 2013, A\&A, 554, A103

Pineda J. L., Langer W. D., Goldsmith P. F., 2014, A\&A, 570, A121

Pineda J. L. et al., 2017, ApJ, 839, 107

Pinto C., Kaastra J. S., Costantini E., Verbunt F., 2010, A\&A, 521, A79

Pinto C., Kaastra J. S., Costantini E., de Vries C., 2013, A\&A, 551, A25

Rauch T., 2016, in Deustua S., Allam S., Tucker D., Smith J. A., eds, ASP Conf. Ser. 503, San Francisco, p.193

Rauch T., Orio M., Gonzales-Riestra R., Nelson T., Still M., Werner K., Wilms J., 2010, ApJ, 717, 363

Richter P. et al., 2017, A\&A, 607, A48

Robin A. C., Reylé C., Derrière S., Picaud S., 2003, A\&A, 409, 523

Savage B. D. et al., 2017, ApJS, 232, 25

Schulz N. S., Corrales L., Canizares C. R., 2016, ApJ, 827, 49

Schwarz G. J. et al., 2011, ApJS, 197, 31

Shannon M. J., Peeters E., Cami J., Blommaert J. A. D. L., 2018, ApJ, 855, 32

Ueda Y., Mitsuda K., Murakami H., Matsushita K., 2005, ApJ, 620, 274

van Rossum D. R., 2012, ApJ, 756, 43

Welty D. E., Lauroesch J. T., Wong T., York D. G., 2016, ApJ, 821, 118

Yao Y., Schulz N. S., Gu M. F., Nowak M. A., Canizares C. R., 2009, ApJ, 696, 1418

This paper has been typeset from a $\mathrm{T}_{\mathrm{E}} \mathrm{X} / \mathrm{L} \mathrm{T} \mathrm{E} \mathrm{X}$ file prepared by the author. 